Pacific Journal of Mathematics

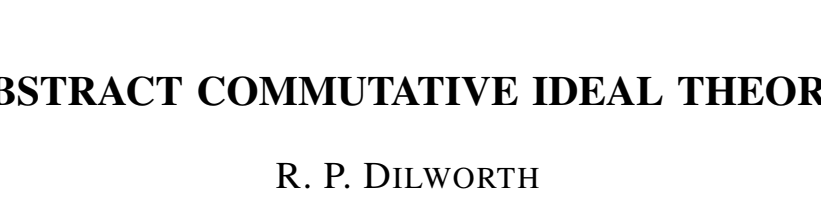




\title{
ABSTRACT COMMUTATIVE IDEAL THEORY
}

\author{
R. P. Dilworth
}

1. Introduction. Several years ago, M. Ward and the author [4] began a study in abstract form of the ideal theory of commutative rings. Since it was intended that the treatment should be purely ideal-theoretic, the system which was chosen for the study was a lattice with a commutative multiplication. For such multiplicative lattices, analogues of the Noether decomposition theorems for commutative rings were formulated and proved. However the theorems corresponding to the deeper results on the ideal structure of commutative rings were not obtained; the essential difficulty being the problem of formulating abstractly the notion of a principal ideal. This difficulty occurred in a mild form in treating the Noether theorem on decompositions into primary ideals. In fact, in the above mentioned paper, a weak concept of "principal element" was introduced which sufficed for the proof of the decomposition theorem into primaries. Nevertheless, the definition had serious defects and it was immediately obvious that it was not adequate for the further development of the abstract theory.

In this paper, I give a new and stronger formulation for the notion of a "principal element", and, in terms of this concept, prove an abstract version of the Krull Principal Ideal Theorem. Since there are generally many non-principal ideals of a commutative ring which are "principal elements" in the lattice of ideals, the abstract theorem represents a considerable strengthening of the classical Krull result.

It seems appropriate at this point to include a brief description of the new "principal elements" and to sketch their relationship to principal ideals.

Let $L$ be a lattice with a multiplication and an associated residuation. The product of two lattice elements $A$ and $B$ will be denoted by $A B$ and the residual, by $A: B$. An element $M$ of $L$ is said to be meet principal if

$$
(A \cap B: M) M \supseteq A M \cap B
$$

all $A, B \in L$.

Similarly, $M$ is said to be join principal if

$$
(A \cup B M): M \subseteq A: M \cup B
$$

all $A, B \in L$.

Finally, $M$ is said to be principal if it is both meet and join principal.

Now let $L$ be the lattice of ideals of a commutative ring $R$ and let $M=(m)$ be a principal ideal of $R$. If $x \in A M \cap B$, then $x \in B$ and

Received April 20, 1961. 
$x=a m$ where $a \in A$. It follows that $a \in B: M$ and hence that $a \in A \cap B: M$. Thus $x=a m \in(A \cap B: M) M$. According to (1.1), $M$ is meet principal. Next let $y \in(A \cup B M): M$. Then $y m \in A \cup B M$ and hence $y m=a+b m$ where $a \in A$ and $b \in B$. Thus $a=(y-b) m$, $y-b \in A: M$, and hence $y=(y-b)+b \in A: M \cup B$. It follows that $M$ is also join principal. Hence every principal ideal is indeed a principal element in the lattice of ideals.

It will be shown in $\$ 7$ that in polynomial rings, properties (1.1) and (1.2) in fact characterize the principal ideals. In more general rings there may be many nonprincipal ideals which are principal elements of the lattice of ideals. Thus if $R$ is the ring of integers in an algebraic number field, then $A: B$ is the quotient $(A \cap B) / B$ and every ideal satisfies (1.1) and (1.2). We note that since distinct prime ideals of $R$ are noncomparable, the conclusion of the Krull Principal Ideal Theorem also holds for all ideals in this ring.

2. Preliminary definitions and results. Throughout the paper, elements of a multiplicative lattice will be denoted by latin capitals, $U$ and $\cap$ will denote the lattice operations and $\supseteqq$ will denote the latice inclusion relation with $\supset$ being reserved for proper inclusion. The lattices to be studied will be complete with unit element $I$ and with null element 0 .

A lattice is said to be multiplicative if there is defined on the lattice a commutative, associative, join distributive, multiplication. We shall also require that $I$ be the identity element for multiplication. Such a multiplicative lattice also has a residuation satisfying the basic relations

$$
\begin{aligned}
& A \supseteqq(A: B) B \\
& A \supseteqq X B \text { implies } A: B \supseteqq X .
\end{aligned}
$$

Further important properties of the residuation and multiplication are the following

(2.11) $(A \cap B): B=A: B$

(2.12) $A:(A \cup B)=A: B$

(2.13) $A:(B \cup C)=A: \mathrm{B} \cap A: C$ 
(2.14) If $A \cup C=B \cup C=I$, then $(A B \cup C)=I$

(2.15) If $A \cup C=I$, then $(A \cap B) \cup C=B \cup C$

(2.16) $\quad\left(A_{1} \cup \cdots \cup A_{n}\right)^{k_{1}+\cdots+k_{n}} \leqq A_{1}^{k_{1}} \cup \cdots \cup A_{n}^{k_{n}}$.

Now let $L$ denote a multiplicative lattice satisfying the ascending chain condition. An element $P \in L$ is prime if the following condition is satisfied for all $A, B \in L$

$$
P \supseteqq A B \text { implies } P \supseteqq A \text { or } P \supseteqq B \text {. }
$$

The element $I$ is trivially a prime according to this definition. However, "prime" will normally refer to prime elements distinct from $I$.

An element $Q \in L$ is primary if for all $A, B \in L$

$$
Q \supseteqq A B \text { implies } Q \supseteqq A \text { or } Q \supseteqq B^{k} \text { for some integer } k \text {. }
$$

Many of the results relating prime and primary ideals in a commutative ring carry over directly to multiplicative lattices. Thus if $Q$ is a primary element of $L$, the join $P_{Q}$ of all elements $X$ such that $X^{s} \cong Q$ for some integer $s$ is a prime element containing $Q$. It is easily verified that $P_{Q}$ is the minimal prime containing $Q$. Furthermore, $Q \supseteqq P_{Q}^{k}$ for some integer $k$. The prime $P_{Q}$ is called the prime associated with $Q$ and it is characterized by the properties

$$
\begin{gathered}
P_{Q} \supseteq Q \supseteqq P_{Q}^{k} \quad \text { for some integer } k \\
Q \supseteqq A B \text { implies } Q \supseteqq A \text { or } P_{Q} \supseteqq B .
\end{gathered}
$$

If $P_{Q} \nsupseteq A$, it is easily verified that $Q: A=Q$. Also if $Q_{1}$ and $Q_{2}$ are primary elements associated with the same prime $P$, then $Q_{1} \cap Q_{2}$ is likewise a primary element associated with $P$.

An element $A$ is said to have a primary decomposition if there exists primaries $Q_{1}, \cdots, Q_{m}$ such that

$$
A=Q_{1} \cap \cdots \cap Q_{m} .
$$

If superfluous $Q_{i}$ are removed and the primaries associated with the same prime are combined we obtain a reduced primary decompsition in which distinct primaries are associated with distinct primes. Such a primary decomposition is called a normal primary decomposition. The fundamental theorem on primary decompositions is proved exactly as in the commutative ring case and may be stated as follows:

Any two normal decompositions of an element $A$ have the same number of components and the same set of associated primes.

Let $A=Q_{1} \cap \cdots \cap Q_{n}$ be a normal decomposition of $A$ and let $P_{1}, \cdots, P_{n}$ denote the associated primes. $A$ subset (5) of $\left\{P_{1}, \cdots, P_{n}\right\}$ is 
isolated if $P_{i} \in \mathbb{B S}$ implies $P_{j} \in \mathbb{B S}$ whenever $P_{i} \supseteqq P_{j}$. The element $A_{\widetilde{S}}=$ $\cap\left\{Q_{i} \mid P_{i} \in \mathbb{S S}\right\}$ is called an isolated component of $A$. The fundamental result on isolated components is the following:

The element $A \subseteq$ depends only upon $A$ and (S) and not upon the particular normal decomposition.

For let $A=Q_{1}^{\prime} \cap \cdots \cap Q_{n}^{\prime}$ be a second normal decomposition and let $A_{\Im}^{\prime}=\bigcap\left\{Q_{1}^{\prime} \mid P_{i} \in(\mathfrak{S}\}\right.$. If we set $B^{\prime}=\cap\left\{Q_{j}^{\prime} \mid P_{j} \notin \mathbb{S}\right\}$, then $Q_{i} \supseteqq A=$ $A_{\Im}^{\prime} \cap B^{\prime} \supseteqq A_{\Im}^{\prime} B^{\prime}$. Now if $P_{i} \in \mathbb{S}$, then $P_{i} \supseteqq B^{\prime}$ since otherwise $P_{i} \supseteqq Q_{j}^{\prime} \supseteqq P_{j}^{k}$ and hence $P_{i} \supseteqq P_{j}$ so that $P_{j} \in$ BS contrary to $P_{j} \notin$ SS. It follows that $Q_{i} \supseteqq A_{\mathscr{S}}^{\prime}$ for all $i$ such that $P_{i} \in \mathbb{B S}$. Thus $A_{\widetilde{S}}=\cap\left\{Q_{i} \mid P_{i} \in \mathbb{S}\right\} \supseteqq A_{\mathscr{S}}^{\prime}$. Similarly $A_{\overparen{S}}^{\prime} \supseteqq A_{\overparen{S}}$ and hence $A_{\overparen{S}}=A_{\overparen{S}}$.

There are multiplicative lattices satisfying the ascending chain condition in which elements fail to have primary decompositions. ${ }^{1}$ On the other hand it is well known that every element of a lattice satisfying the ascending chain condition has a decomposition into meet irreducibles. Since a meet irreducible can have a primary decomposition only if it is itself primary, it follows that the elements of $L$ will have primary decomposition if and only if every meet irreducible element of $L$ is primary. Suitable condition which insure that meet irreducibles will be primary involve properties of principal elements. These will be discussed in the following section.

It should be pointed out that the relationship between prime and primary elements can be developed in multiplicative lattices which are compactly generated. These include multiplicative lattices satisfying the ascending chain conditions as a very special case.

3. Noether lattices. In $\S 1$, the notions of meet principal elements, join principal elements, and principal elements were introduced. We begin by developing some elementary properties of these elements.

Lemma 3.1 An element $M$ is meet principal if and only if

$$
(A \cap B: M) M=A M \cap B \quad \text { all } A, B \in L \text {. }
$$

For $(A \cap B: M) M \subseteq A M \cap(B: M) M \cong A M \cap B$ by 2.10 and 2.1

LEMmA 3.2 An element $M$ is join principal if and only if

$$
(A \cup B M): M=A: M \cup B \quad \text { all } A, B \in L \text {. }
$$

For $(A \cup B M): M \supseteqq A: M \cup(B M): M \supseteqq A: M \cup B$ by 2.9 and 2.8. Setting $A=I$ in 3.1 and $A=0$ in 3.2 gives the following corollaries

1 Ward-Dilworth [4] contains several examples. 
Corollary 3.1. If $M$ is meet principal, then

$$
(B: M) M=B \cap M \quad \text { all } B \in L \text {. }
$$

Corollary 3.2. If $M$ is join principal, then

$$
(B M): M=B \cup(0: M) \quad \text { all } B \in L .
$$

In particular, if $M \supseteqq B$, then $(B: M) M=B$ and if $B \supseteqq 0: M$, then $(B M): M=B$ respectively.

Lemma 3.3. If $M_{1}$ and $M_{2}$ are meet principal, then $M_{1} M_{2}$ is meet principal.

For

$$
\begin{aligned}
& {\left[A \cap B:\left(M_{1} M_{2}\right)\right] M_{1} M_{2}=\left[A \cap\left(B: M_{2}\right): M_{1}\right] M_{1} M_{2}} \\
& \quad=\left(A M_{1} \cap B: M_{2}\right) M_{2}=A M_{1} M_{2} \cap B
\end{aligned}
$$

by (2.5) and (3.1).

Lemma 3.4. If $M_{1}$ and $M_{2}$ are join principal, then $M_{1} M_{2}$ is join principal.

For

$$
\begin{aligned}
& \left(A \cup B M_{1} M_{2}\right):\left(M_{1} M_{2}\right)=\left[\left(A \cup B M_{1} M_{2}\right): M_{2}\right]: M_{1} \\
& \quad=\left[A: M_{2} \cup B M_{1}\right]: M_{1}=\left(A: M_{2}\right): M_{1} \cup B=A:\left(M_{1} M_{2}\right) \cup B
\end{aligned}
$$

by (2.5) and (3.2).

CoRollary 3.3. If $M_{1}$ and $M_{2}$ are principal, then $M_{1} M_{2}$ is principal. The conditions which insure that every meet irreducible is primary are formulated in the following theorem.

THEOREM 3.1. Let $L$ be a modular, multiplicative lattice satisfying the ascending chain condition. Furthermore let every element of $L$ be a join of meet principal elements. Then every meet irreducible element is primary.

Proof. Let $Q$ be meet irreducible and let $Q \supseteqq A M$ where $Q \nsupseteq A$ and $M$ is meet principal. Then by (2.13) $(A \cup Q): M \cong(A \cup Q): M^{2} \cong$ $\cdots \subseteq(A \cup Q): M^{k} \leqq \cdots$. By the ascending chain condition there exists $k$ such that

$$
(A \cup Q): M^{k}=(A \cup Q): M^{k+1} .
$$


Now let $C=(A \cup Q) \cap\left(M^{k+1} \cup Q\right)$.

Then $C: M^{k+1}=(A \cup Q): M^{k+1} \cap\left(M^{k+1} \cup Q\right): M^{k+1}=(A \cup Q): M^{k+1} \cap I=$ $(A \cup Q): M^{k+1}=(A \cup Q): M^{k}$ by (2.4).

By Lemma $3.3 M^{k}$ and $M^{k+1}$ are meet principal and hence by (3.3) $\left(C: M^{k+1}\right) M^{k+1}=C \cap M^{k+1}$ and $\left[(A \cup Q): M^{k}\right] M^{k}=(A \cup Q) \cap M^{k}$. Thus by modularity we have $C=\left(M^{k+1} \cup Q\right) \cap C=Q \cup\left(C \cap M^{k+1}\right)=$ $Q \cup\left(C: M^{k+1}\right) M^{k+1}=Q \cup\left[(A \cup Q): M^{k}\right] M^{k+1}=Q \cup\left[(A \cup Q) \cap M^{k}\right] M$ $\subseteq Q \cup(A \cup Q) M=Q \cup A M \cup Q M \sqsubseteq Q \sqsubseteq C$.

Hence

$$
Q=C=(A \cup Q) \cap\left(M^{k+1} \cup Q\right) .
$$

Since $Q$ is meet irreducible and $Q \neq A \cup Q$ we must have $Q=M^{k+1} \cup Q$ and hence $Q \supseteqq M^{k+1}$. Now let $Q \supseteqq A B$ where $Q \nsupseteq \mathrm{A}$. Then $B$ is a join of meet principal elements, say $B=M_{1} \cup \cdots \cup M_{r}$. Thus $Q \supseteqq A M_{i}$ for $i=1, \cdots, r$. Hence there exists $k_{i}$ such that $Q \supseteqq M_{i}^{k_{i}}$. Let $k=$ $k_{1}+\cdots+k_{r}$. Then by (2.16) $Q \supseteqq B^{k}$ and it follows that $Q$ is primary.

In Ward-Dilworth [4], a multiplicative lattice satisfying the ascending chain condition in which every irreducible is primary was called a "Noether lattice." Since these conditions are not sufficient for the results of commutative ideal theory we shall strengthen the definition as follows:

Definition 3.1. A multiplicative lattice $L$ is a Noether lattice if the following conditions are satisfied:

(1) $L$ is modular

(2) $L$ satisfies the accending chain condition

(3) Every element of $L$ is a join of principal elements.

From the results of $\$ 1$ we conclude the lattice of ideals of a Noetherian ring is a Noether lattice.

Theorem 3.4 implies that every irreducible element of a Noether lattice is primary. From the results of $\$ 2$, it follows that every element of a Noether lattice has a normal primary decomposition.

Now let $A=Q_{1} \cap \cdots \cap Q_{n}$ be a normal decomposition of $A$ and let $\left\{P_{1}, \cdots, P_{n}\right\}$ be the associated primes. If $B$ is an arbitrary element of $L$, then $\left\{P_{i} \mid P_{i} \cup B \neq I\right\}$ is clearly an isolated set of primes. Let $A_{B}$ denote the corresponding isolated component of $A$. We prove now an abstract version of the intersection theorem.

THEOREM 3.2. (Intersection Theorem) If $A$ and $B$ are elements of a Noether lattice, then $\cap_{k}\left(A \cup B^{k}\right)=A_{B}$.

Proof. According to the definition of isolated components $A_{B}=$ $\cap\left\{Q_{i} \mid P_{i} \cup B \neq I\right\}$. Let $A_{B}^{*}=\cap\left\{Q_{j} \mid P_{j} \cup B=I\right\}$. Now $P_{j} \cup B=I$ 
implies $P_{j}^{k} \cup B=I$ for all $k$ by (2.14) and hence $Q, \cup B=I$. But then

$$
A_{B}^{*}=\cap\left\{Q_{j} \mid P_{j} \cup B=I\right\} \supseteqq I\left\{Q_{j} \mid P_{j} \cup B=I\right\}
$$

and hence $A_{B}^{*} \cup B=I$ by (2.14). From (2.14) we get $A_{B}^{*} \cup B^{k}=I$ all $k$. Hence $A \cup B^{k}=\left(A_{B} \cap A_{B}^{*}\right) \cup B^{k}=A_{B} \cup B^{k}$ by 2.15. Thus $A \cup B^{k} \supseteqq A_{B}$ all $k$ and hence $\cap_{k}\left(A \cup B^{k}\right) \supseteqq A_{B}$.

Next let $\cap_{k}\left(A \cup B^{k}\right) \supseteqq M$ where $M$ is principal.

Let $A \cup B M=R_{1} \cap \cdots \cap R_{m}$ be a normal decomposition of $A \cup B M$ with associated primes $P_{1}^{\prime}, \cdots P_{m}^{\prime}$. Then $R_{i} \supseteqq B M$ and hence either $R_{i} \supseteqq M$ or $P_{i}^{\prime} \supseteqq B$. But if $P_{\imath}^{\prime} \supseteqq B$, then $R_{i} \supseteqq P_{i}^{\prime k_{i}} \supseteqq B^{k_{i}}$ and hence $R_{i} \supseteqq A \cup B^{k_{i}} \supseteqq \cap_{k}\left(A \cup B^{k}\right) \supseteqq M$. In either case we get $R_{i} \supseteqq M$ and hence $A \cup B M=R_{1} \cap \cdots \cap R_{m} \supseteqq M$. It follows that $A \cup B M=A \cup M$. Since $M$ is principal we have

$$
A: M \cup B=(A \cup B M): M=(A \cup M): M=I .
$$

Thus $P_{i} \cup B \neq I$ implies $P_{i} \nsupseteq A: M$. Since $Q_{i} \supseteqq A \supseteqq(A: M) M$ it follows that $Q_{i} \supseteqq M$ for all $i$ such that $P_{i} \cup B \neq I$. Hence $A_{B}=$ $\cap\left\{Q_{i} \mid P_{i} \cup B \neq I\right\} \supseteqq M$.

Since $L$ is a Noether lattice, $\cap_{k}\left(A \cup B^{k}\right)$ is a join of principal elements. Thus

$$
A_{B} \supseteq \bigcap_{k}\left(A \cup B^{k}\right)
$$

and the proof of the theorem is complete

Setting $A=0$ in Theorem 3.2 we get

CoRollary 3.1. If $B$ is an element of a Noether lattice $L$, then

$$
\cap B^{k}=0_{B} \text {. }
$$

If $A \neq I$ is an element of a Noether lattice, then there exists a maximal element $P \neq I$ such that $P \supseteqq A$. By (2.14) and (2.17) such an element is prime and clearly is a maximal prime of $L$.

Definition 3.2. A Noether lattice $L$ is local if it contains precisely one maximal prime.

Now suppose that $L$ is local with the unique maximal prime $P_{0}$. If $P_{i} \neq I$ and $B \neq I$, then $P_{0} \supseteqq P_{i} \cup B$ and hence $P_{i} \cup B \neq I$. Thus $A_{B}=\cap\left\{Q_{i} \mid P_{i} \cup B \neq I\right\}=A$. Accordingly we get

Corollary 3.2. If $L$ is a local Noether lattice and $B \neq I$ is an element of $L$, then for each $A \in L$

$$
\bigcap_{k}\left(A \cup B^{k}\right)=A \text {. }
$$

Setting $A=0$, we have 

then

CoRollary 3.3. If $B \neq I$ is an element of a local Noether lattice,

$$
\bigcap_{k} B^{k}=0
$$

4. Quotient lattices. If $L$ is a Noether lattice and $D \in L$, then $L / D=\{A \in L \mid A \supseteqq D\}$ is clearly a sublattice of $L$. However, $L / D$ need not be closed with respect to multiplication. We make $L / D$ into a multiplicative lattice by defining

$$
A \circ B=A B \cup D \text {. }
$$

This multiplication is easily seen to be commutative, associative, distributive with respect to join, and has $I$ as an identity element. Thus with this multiplication $L / D$ is a multiplicative lattice.

Since $A: B \supseteqq A$, it follows that $L / D$ is closed with respect to residuation. This residuation is also the residuation associated with $A \circ B$ since

$$
A \supseteqq X \circ B \text { if and only if } A \supseteqq X B \text {. }
$$

Lemma 4.1. If $L$ is a Noether lattice, then $L / D$ is a Noether lattice. Furthermore if $M$ is a principal element of $L$, then $M \cup D$ is a principal element of $L / D$.

For if $L$ is modular and satisfies the ascending chain condition, then clearly $L / D$ is modular and satisfies the ascending chain condition.

Now let $M$ be a principal element of $L$ and let $A, B \in L / D$. Then

$$
\begin{aligned}
{[A \cup B \circ(M \cup D)]:(M \cup D) } & =(A \cup B M \cup D):(M \cup D) \\
& =(A \cup B M \cup D): M=(A \cup B M): M \\
& =A: M \cup B=A:(M \cup D) \cup B
\end{aligned}
$$

and $M \cup D$ is thus join principal. On the other hand

$$
\begin{aligned}
{[A} & :(M \cup D) \cap B] \circ(M \cup D)=(A: M \cap B) \circ(M \cup D) \\
& =(A: M \cap B)(M \cup D) \cup D \\
& =(A: M \cap B) M \cup(A: M \cap B) D \cup D=(A: M \cap B) M \cup D \\
& =(A \cap B M) \cup D=A \cap(B M \cup D) \\
& =(A \cap(B M \cup B D) \cup D)=A \cap B \circ(M \cup D) .
\end{aligned}
$$

and $M \cup D$ is thus meet principal. Note that modularity is required in the sixth step of this argument. Now if $A \in L / D$, then $A=A \cup D=$ $M_{1} \cup \cdots \cup M_{k} \cup D=\left(M_{1} \cup D\right) \cup \cdots \cup\left(M_{k} \cup D\right)$ and hence every element of $L / D$ is a join of principal elements. Thus $L / D$ is a Noether lattice. 
The next lemma shows that the arithmetical properties of $L$ are preserved in $L / D$.

Lemma 4.2. An element is prime or primary in $L / D$ if and only if it is prime or primary in $L$.

Let $A, B, C \in L / D$. Then $A \supseteq B \circ C$ if and only if $A \supseteq B C$. Hence $A$ is prime or primary if and only if it is prime or primary in $L$.

Corollary 4.1. If $L$ is a local Noether lattice, and $D \neq I$, then $L / D$ is a local Noether lattice.

5. Congruence lattices. Congruence relations provide a second method for constructing new Noether lattices from a given Noether lattice. This construction is the abstract version of the construction of the generalized ring of quotients introduced by Chevalley [1]. Let $L$ be a Noether lattice and let $D$ be an arbitrary element of $L$. If $P_{1}, \cdots, P_{n}$ are the primes associated with a normal decompsition of $A$, then $\left\{P_{i} \mid D \supseteqq P_{i}\right\}$ is an isolated set of primes and hence determines an isolated component $A_{D}$ of $A$. For a given normal decomposition $A=Q_{1} \cap \cdots \cap Q_{n}$ we have $A_{D}=\cap\left\{Q_{i} \mid D \supseteqq P_{i}\right\}$. We will let $A_{D}^{\prime}$ denote $\left\{Q_{j} \mid D \nsupseteq P_{j}\right\}$ so that $A=A_{D} \cap A_{D}^{\prime}$. We define $A \equiv B(D)$ if and only if $A_{D}=B_{D}$.

\section{LEMma 5.1. If $A \supseteqq B, A_{D} \supseteqq B_{D}$.}

For let $A=Q_{1} \cap \cdots \cap Q_{m}$ be a normal decomposition of $A$ and let $P_{1}, \cdots, P_{m}$ be the associated primes. If $D \supseteqq P_{i}$, then $Q_{i} \supseteqq A \supseteqq B \supseteqq$ $B_{D} \cap B_{D}^{\prime}$. If $P_{i} \supseteqq B_{D}^{\prime}$, then $P_{i} \supseteqq P_{j}^{\prime}$ where $D \nsupseteq P_{j}^{\prime}$ contrary to $D \supseteqq P_{i}$. Hence $P_{i} \nsupseteq B_{D}^{\prime}$ and thus $Q_{i} \supseteqq B_{D}$. It follows that $A_{D}=\cap\left\{Q_{i} \mid D \supseteqq P_{i}\right\} \supseteqq B_{D}$.

Lemma 5.2. If $A \equiv B(D)$, then $A \cap C \equiv B \cap C(D)$.

For let $A \cap C=Q_{1} \cap \cdots \cap Q_{n}$ be a normal decomposition of $A \cap C$ with associated primes $P_{1}, \cdots P_{n}$. Then if $D \supseteqq P_{i}$ we have $Q_{i} \supseteqq A \cap C=$ $A_{D} \cap C \cap A_{D}^{\prime}$ and $P_{i} \nsupseteq A_{D}^{\prime}$. Thus $Q_{i} \supseteqq A_{D} \cap C=B_{D} \cap C \supseteqq B \cap C$. Thus $(A \cap C)_{D}=\cap\left\{Q_{i} \mid D \supseteqq P_{i}\right\} \supseteqq B \cap C$. But then $(A \cap C)_{D}=\left((A \cap C)_{D}\right)_{D} \supseteqq$ $(B \cap C)_{D}$ by Lemma 5.1. Similarly $(B \cap C)_{D} \supseteq(A \cap C)_{D}$ and thus $(A \cap C)_{D}=(B \cap C)_{D}$. It follows that $A \cap C \equiv B \cap C(D)$.

Lemma 5.2. If $A \equiv B(D)$, then $A \cup C \equiv B \cup C(D)$.

For let $A \cup C=Q_{1} \cap \cdots \cap Q_{n}$ be a normal decomposition of $A \cup C$ with associated primes $P_{1}, \cdots, P_{n}$. Then if $D \supseteqq P_{i}$ we have $Q_{i} \supseteqq$ $A_{D} \cap A_{D}^{\prime}$ and $Q_{i} \supseteqq C$. Since $P_{i} \nsupseteq A_{D}^{\prime}$ we have $Q_{i} \supseteqq A_{D}$. Thus $Q_{i} \supseteqq B_{D} \supseteqq B$ and this $Q_{i} \supseteqq B \cup C$. But then $(A \cup C)_{D}=\cap\left\{Q_{i} \mid D \supseteqq P_{i}\right\} \supseteqq B \cup C$ and hence $(A \cup C)_{D} \supseteq(B \cup C)_{D}$ by Lemma 5.1. Similarly $(B \cup C)_{D} \supseteq(A \cup C)_{D}$ and hence $A \cup C \equiv B \cup C(D)$. 
Lemma 5.3. If $A \equiv B(D)$, then $A C \equiv B C(D)$.

For let $A C=Q_{1} \cap \cdots \cap Q_{n}$ be a normal decomposition of $A C$ with associated primes $P_{1}, \cdots, P_{n}$. Then if $D \supseteqq P_{i}$ we have $Q_{i} \supseteqq A C=$ $\left(A_{D} \cap A_{D}^{\prime}\right) C \supseteqq A_{D} C A_{D}^{\prime}$ where $P_{i} \nsupseteq A_{D}^{\prime}$. Thus $Q_{i} \supseteqq A_{D} C=B_{D} C \supseteqq B C$. It follows that $(A C)_{D} \supseteqq B C$ and hence by Lemma 5.1 that $(A C)_{D} \supseteqq(B C)_{D}$. Similarly $(B C)_{D} \supseteqq(A C)_{D}$ and hence the conclusion of the lemma follows.

Lemma 5.4. If $A \equiv B(D)$, then $A: C \equiv B: C(D)$ and $C: A \equiv C: B(D)$.

For let $X: Y=Q_{1} \cap \cdots \cap Q_{n}$ be a normal decomposition of $X: Y$ with associated primes $P_{1}, \cdots, P_{n}$. Then if $D \supseteqq P_{i}$ we have $Q_{i} \supseteqq X: Y=$ $\left(X_{D} \cap X_{D}^{\prime}\right): Y=\left(X_{D}: Y\right) \cap\left(X_{D}^{\prime}: Y\right) \supseteqq\left(X_{D}: Y\right)\left(X_{D}^{\prime}: Y\right)$. Now $P_{i} \nsupseteq X_{D}^{\prime}: Y$ since otherwise $P_{i} \supseteqq X_{D}^{\prime}: Y \supseteqq X_{D}^{\prime}$. Thus $Q_{i} \supseteqq X_{D}: Y \supseteqq X_{D}: Y_{D}$ since $Y_{D} \supseteqq Y$. Hence $(X: Y)_{D}=\cap\left\{Q_{i} \mid D \supseteq P_{i}\right\} \supseteqq X_{D}: Y_{D}$.

On the other hand, $X \supseteqq(X: Y) Y$ and hence

$$
X_{D} \supseteqq[(X: Y) Y]_{D}=(X: Y)_{D} Y_{D} \text { by Lemma } 5.1 \text { and } 5.3 \text {. }
$$

Hence $X_{D}: Y_{D} \supseteqq(X: Y)_{D}$ and thus $(X: Y)_{D}=X_{D}: Y_{D}$ by the result of the previous paragraph.

Now if $A \equiv B(D)$, then $(A: C)_{D}=A_{D}: C_{D}=B_{D}: C_{D}=(B: C)_{D}$ and thus $A: C=B: C(D)$. Similarly $C: A \equiv C: B(D)$.

Lemma 5.1-5.4 show that congruence $\bmod D$ is a congruence relation on $L$ preserving meet, join, multiplication, and residuation. In view of the fact that principal elements are defined in terms of equations involving meet, join, multiplication, and residuation it follows that the congruence class of a principal element is principal in the lattice of congruence classes.

Now let $L_{D}$ denote the multiplicative lattice of congruence classes. Then we have

THEOREM 5.1. If $L$ is a Noether lattice, then $L_{D}$ is a Noether lattice.

The arithmetical properties of $L_{D}$ may be described as follows:

THEOREM 5.2. The primes and primaries of $L_{D}$ are precisely the congruence classes determined by the primes and primaries of $L$. The proper prime elements of $L_{D}$ are the congruence classes determined by primes $P$ such that $D \supseteqq P$.

Proof. Let the congruence class $\{C\}$ be a prime in $L_{D}$. Now $C \supseteqq P_{1}^{k_{1}} \cdots P_{n}^{k_{n}}$ where $P_{1}, \cdots, P_{n}$ are primes of $L$ such that $P_{i} \supseteqq C$. But then $\{C\} \supseteqq\left\{P_{1}\right\}^{k_{1}} \cdots\left\{P_{n}\right\}^{k_{n}}$ and hence $\{C\} \supseteqq\left\{P_{i}\right\} \supseteqq\{C\}$ for since $i$. Thus $\{C\}=\left\{P_{i}\right\}$ where $P_{i}$ is a prime of $L$. Conversely, if $P$ is a prime of $L$, then, by definition, $P_{D}=P$ or $P_{D}=I$ according as $D \supseteqq P$ or $D \nsupseteq P$. Hence $\{P\} \supseteqq\{A\}\{B\}$ implies $P_{D} \supseteqq A_{D} B_{D}$ and hence $P_{D} \supseteqq A_{D}$ or 
$P_{D} \supseteqq B_{D}$ which implies $\{P\} \supseteqq\{A\}$ or $\{P\} \supseteqq\{B\}$. Thus the primes of $L_{D}$ have the form $\{P\}$ where $P$ is a prime of $L$ and $\{P\}$ is proper if and only if $D \supseteqq P$.

Next let $\{C\}$ be a primary element of $L_{D}$ associated with the prime $\{P\}$. Now $C_{D}=Q_{1} \cap \cdots \cap Q_{r}$ when $D \supseteqq P_{i}$ and $P_{i}$ is the prime associated with $Q_{i}$. We may suppose that $\{C\} \neq\{I\}$ and thus that $D \supseteqq P$. Now $\{C\} \supseteqq\{P\}^{k}$ for some $k$. Hence $C_{D} \supseteqq P_{D}^{k}=P^{k}$. But then $P_{i} \supseteqq P$ for all $i$. Since $P \supseteqq C_{D}$ we have $P \supseteqq P_{j}$ for some $j$ and hence $P=P_{j}$. On the other hand $P_{1}, \cdots, P_{r}$ are distinct and thus $P \nsupseteq P_{i}$ for $i \neq j$. Since $\{C\} \supseteqq\left\{Q_{1}\right\} \cdots\left\{Q_{r}\right\}$ and $\{P\} \nsupseteq\left\{Q_{i}\right\}$ for $i \neq j$. We have $\{C\} \supseteqq\left\{Q_{j}\right\}$ since $\{C\}$ is primary in $L_{o}$. But then $\{C\}=\left\{Q_{j}\right\}$ where $Q_{j}$ is a primary of $L$. Conversely if $Q$ is a primary element of $L$, then, by definition, $Q_{D}=Q$ or $Q_{D}=I$ according as $D \supseteqq P_{Q}$ or $D \nsupseteq P_{Q}$. Hence $\{Q\} \supseteqq\{A\}\{B\}$ implies $\{Q\} \supseteqq\{A\}$ or $\{P\} \supseteqq\{B\}$ and $\{Q\}$ is a primary element of $L_{D}$. This completes the proof of the theorem.

It should be noted that distinct primes contained in $D$ determine distinct congruence classes.

TheOREM 5.3. If $P$ is a proper prime of $L$, then $L_{P}$ is a local Noether lattice.

Proof. For if $\left\{P^{\prime}\right\}$ is a proper prime of $L_{P}$, then $P \supseteqq P^{\prime}$ and hence $\{P\} \supseteqq\left\{P^{\prime}\right\}$. On the other hand $\{P\}$ is a proper prime of $L_{P}$. Thus $\{P\}$ is the unique maximal proper prime of $L_{P}$.

6. The principal element theorem. The critical steps in the proof of the principal element theorem will depend upon the finite dimensionality of certain quotient lattices. The finite dimensionality rests in turn upon the following theorem on modular lattices.

THEOREM 6.1. Let $S$ be a subset of a modular lattice $L$ which generates $L$ under finite joins. Then if $S$ satisfies the descending chain condition, $L$ also satisfies the descending chain condition. lattice.

We begin by proving two lemmas on quotient lattices in a modular

Lemma 6.1. Let $L$ be a modular lattice. If $a / b$ and $b / c$ are quotient lattices satisfying the descending chain condition, then a/c satisfies the descending chain condition.

For let $x_{1} \geqq x_{2} \geqq \cdots \geqq x_{n} \geqq \cdots$ be a descending chain in $a / c$. Then $x_{1} \cup b \geqq x_{2} \cup b \geqq \cdots \geqq x_{n} \cup b \geqq \cdots$ and $x_{1} \cap b \geqq x_{2} \cap b \geqq \cdots \geqq x_{n} \cap b \geqq \cdots$ are descending chains in $a / b$ and $b / c$ respectively. Hence there exists an integer $k$ such that $x_{n} \cup b=x_{k} \cup b$ and $x_{n} \cap b=x_{k} \cap b$ all $n \geqq k$. 
Since $x_{k} \geqq x_{n}$ all $n \geqq k$ it follows from the modular law that $x_{k}=x_{n}$ all $n \geqq k$ and hence $a / c$ satisfies the descending chain condition.

LEMMA 6.2. Let $L$ be a modular lattice. If $a / b$ and $c / d$ satisfy the descending chain condition, then $a \cup c / b \cup d$ and $a \cap c / b \cap d$ satisfy the descending chain condition.

For $a / a \cap(b \cup d)$ is a subquotient of $a / b$ and hence satisfies the descending chain condition. But $a / a \cap(b \cup d)$ is isomorphic to $a \cup d / b \cup d$ and hence $a \cup d / b \cup d$ satisfies the descending chain condition. A similar argument shows that $a \cup c / a \cup d$ satisfies the descending chain condition. But then $a \cup c / b \cup d$ satisfies the descending chain condition by Lemma 6.1. A dual proof shows that $a \cap c / b \cap d$ likewise satisfies the descending chain condition.

Repeated application of Lemma 6.2 gives the following corollary.

COROLlaRY 6.1. Let $L$ be a modular lattice. If $a_{1} / b, \cdots, a_{n} / b$ satisfy the descending chain condition, then $\left(a_{1} \cup \cdots \cup a_{n}\right) / b$ satisfies the descending chain condition.

We now give the proof of the theorem.

Proof of Theorem 6.1. If $s_{1}$ and $s_{2}$ are elements of $S$, then $s_{1} \cap s_{2}$ is a join of elements of $S$. Hence there exists $s_{3} \in S$ such that $s_{1} \geqq s_{3}$ and $s_{2} \geqq s_{3}$. Thus $S$ is a directed set and hence has a null element $z$ by the descending chain condition. $z$ is clearly the null element of $L$. We shall show first that the quotient lattices $s / z$ satisfy the descending chain condition by making an induction upon the elements of $S$. Clearly $z / z$ satisfies the descending chain condition. Let us suppose that $s / z$ satisfies the descending chain condition for all $s<s_{0}$. If $s_{0} / z$ does not satisfy the descending chain condition, there exists an infinite descending chain

$$
a_{0}>a_{1}>\cdots>a_{n}>\cdots
$$

in $s_{0} / z$. Then $s_{0} \geqq a_{0}>a_{1}$ and $a_{1}=s_{1} \cup \cdots \cup s_{n}$. But then $s_{i} / z$ satisfies the descending chain condition by the induction assumption and hence $a_{1} / z=\left(s_{1} \cup \cdots \cup s_{n}\right) / z$ satisfies the descending chain condition by Corollary 6.1. But $a_{1}>\cdots>a_{n}>\cdots$ is an infinite descending chain in $a_{1} / z$. Hence $s_{0} / z$ satisfies the descending chain condition. Since $S$ itself satisfies the descending chain condition it follows by induction that $s / z$ satisfies the descending chain condition for all $s \in S$. Now let $a \in L$ and let $a=$ $s_{1} \cup \cdots \cup s_{m}$. Since $s_{i} / z$ satisfies the descending chain condition for each $i$, it follows from Corollary 6.1 that $a / z$ satisfies the descending chain condition and hence that $L$ itself satisfies the descending chain condition. This completes the proof of the theorem. 
The following theorem describes the finite dimensionality properties of local Noether lattices.

TheOREM 6.2. Let $L$ be a local Noether lattice with maximal prime $P$. Then $L / P^{k}$ is finite dimensional.

Proof. The elements $P^{k} \cup M$ where $M$ is principal generate $L / P^{k}$ under finite joins. Hence by Theorem 6.1 it will suffice to show that these elements satisfy the descending chain condition. Let

$$
P^{k} \cup M_{1} \supset P^{k} \cup M_{2} \supset \cdots \supset P^{k} \cup M_{n} \cdots
$$

We may clearly assume that $P^{k} \cup M_{1} \neq I$. Since $L$ has exactly one maximal prime element $P$ it follows that $P \supseteqq P^{k} \cup M_{1} \supseteqq M_{1}$. Now $\left(P^{k} \cup M_{r+1}\right): M_{r} \neq I$ since otherwise $M_{r}=\left[\left(P^{k} \cup M_{r+1}\right): M_{r}\right] M_{r}=$ $\left(P^{k} \cup M_{r+1}\right) \cap M_{r}$ by Corollary 3.1 and hence $P^{k} \cup M_{r+1} \supseteqq M_{r}$ which implies $P^{k} \cup M_{r+1} \supseteqq P^{k} \cup M_{r}$ contrary to $P^{k} \cup M_{r} \supset P^{k} \cup M_{r+1}$. Thus

$$
P \supseteqq\left(P^{k} \cup M_{r+1}\right): M_{r} \text { for all } r \text {. }
$$

Now $P \supseteqq M_{1}$. Suppose that $P^{r} \supseteqq M_{r}$ where $r<k$. Then

$$
\begin{aligned}
P^{r+1} \supseteqq\left[\left(P^{k} \cup M_{r+1}\right): M_{r}\right] P^{r} \supseteqq\left[\left(P^{k} \cup M_{r+1}\right): M_{r}\right] M_{r} \\
\\
=\left(P^{k} \cup M_{r+1}\right) \cap M_{r} .
\end{aligned}
$$

Thus $P^{r+1} \supseteqq P^{k} \cup\left[\left(P^{k} \cup M_{r+1}\right) \cap M_{r}\right]=\left(P^{k} \cup M_{r+1}\right) \cap\left(P^{k} \cup M_{r}\right)=$ $P^{k} \cup M_{r+1} \supseteqq M_{r+1}$ by the modular law. Hence by induction we get

$$
P^{k} \supseteqq M_{k} \text {. }
$$

Thus $P^{k}=P^{k} \cup M_{k} \supset P^{k} \cup M_{k+1} \supseteqq P^{k}$ which is impossible. It follows that the elements $P^{k} \cup M$ satisfy the descending chain condition and hence $L / P^{k}$ is finite dimensional.

A lemma on lattice isomorphism will be needed in the proof of the main theorem.

Lemma 6.3. Let $L$ be a Noether lattice in which 0 is a prime. Then if $M$ is a nonzero principal element the quotient lattice $A M / B M$ is lattice isomorphic to $A / B$.

For if 0 is a prime, then $0 \supseteqq(0: X) X$ where $X \neq 0$ implies $0 \supseteqq 0: X$. Hence $0: X=0$ whenever $X \neq 0$. But then $(X M): M=(0 \cup X M): M=$ $0: M \cup X=X$. Now the mapping $X \rightarrow X M$ maps $A / B$ into $A M / B M$. It is order preserving since $X \supseteqq Y$ implies $X M \supseteqq Y M$. It is 1-1 since $X M=Y M$ implies $X=(X M): M=(Y M): M=Y$. Finally if $V \in A M / B M$, then $A M \supseteqq V$ and hence $V=V \cap A M=(V: M \cap A) M$ since $M$ is principal. But $A \supseteqq A \cap V: M \supseteqq A \cap(B M): M \supseteqq A \cap B=B$ and hence 
$X=A \cap V: M$ maps onto $V$. Thus $X \rightarrow X M$ is a $1-1$ order preserving map of $A / B$ onto $A M / B M$ and hence $A / B$ and $A M / B M$ are lattice isomorphic:

In formulating the proof of the Principal Element Theorem, we will make use of a modification of an argument due to D. Rees [3].

THEOREM 6.3. Let $L$ be a local Noether lattice in which 0 is a prime. Let $P$ be the maximal prime of $L$. Then if the principal element $M$ is primary with associated prime $P, P$ is the only non zero prime of $L$.

Proof. Let $P^{\prime}$ be a nonzero prime of $L$. Then $P^{\prime} \supseteqq N$ where $N$ is a nonzero principal element of $L$. Now by (2.13)

$$
N: M \sqsubseteq N: M^{2} \subseteq \cdots \sqsubseteq N: M^{n} \subseteq \cdots .
$$

Since the ascending chain condition holds in $L$, it follows that $N: M^{k}=$ $N: M^{k+1}$ for some $k$. But then

$$
N \cap M^{k+1}=\left(N: M^{k+1}\right) M^{k+1}=\left(N: M^{k}\right) M^{k} M=\left(N \cap M^{k}\right) M .
$$

Then if $d(A / B)$ denotes the dimension of the quotient $A / B$ we have

$$
\begin{aligned}
d\left(\left(N \cup M^{k+1}\right) / M^{k+1}\right) & =d\left(N /\left(N \cap M^{k+1}\right)\right) \\
& =d\left(N /\left(N \cap M^{k}\right) M\right) \\
& =d(N / N M)+d\left(N M /\left(N \cap M^{k}\right) M\right) \\
& =d(I / M)+d\left(N /\left(N \cap M^{k}\right)\right) \\
& =d\left(M^{k} / M^{k+1}\right)+d\left(\left(N \cup M^{k}\right) / M^{k}\right) \\
& =d\left(\left(N \cup M^{k}\right) / M^{k+1}\right)
\end{aligned}
$$

making use of modularity and Lemma 6.3. Note that all of the quotients are finite dimensional since $M \supseteqq P^{l}$ for some $l$ and hence $M^{k+1} \supseteqq P^{(k+1) l}$. But $L / P^{(k+1) l}$ is finite dimensional by Theorem 6.2. Since $N \cup M^{k} \supseteqq$ $N \cup M^{k+1}$ it follows from the equality of the dimension that $N \cup M^{k}=$ $N \cup M^{k+1}$.

Then

$$
\begin{aligned}
M^{k}=M^{k} \cap\left(N \cup M^{k}\right) & =M^{k} \cap\left(N \cup M^{k+1}\right)=\left(M^{k} \cap N\right) \cup M^{k+1} \\
& =\left(N: M^{k}\right) M^{k} \cup M^{k+1}=\left(N: M^{k} \cup M\right) M^{k} .
\end{aligned}
$$

Hence $I=M^{k}: M^{k}=\left[\left(N: M^{k} \cup M\right) M^{k}\right]: M^{k}=N: M^{k} \cup M$. Now $P \nsupseteq$ $N: M^{k}$ since otherwise $P \supseteqq N: M^{k} \cup M=I$. Since $L$ is local with maximal prime $P$ it follows that $N: M^{k}=I$ and hence $N \supseteqq M^{k}$. But then

$$
P^{\prime} \supseteqq N \supseteqq M^{k} \supseteqq P^{k l}
$$


Thus $P^{\prime} \supseteqq P$ and since $P$ is maximal we have $P^{\prime}=P$. This completes the proof of the theorem.

Definition 6.1. Let $P$ be a prime element of a Noether lattice $L$. The least upper bound of the length of chains $P \supset P_{1} \supset P_{2} \supset \cdots \supset P_{k}$ where the $P_{i}$ are primes of $L$ is called the rank of $P$.

The abstract version of the Krull Principal Ideal Theorem (Krull [2]) is formulated as follows:

THEOREM 6.4. (Principal Element Theorem) Let $P$ be a minimal prime containing the principal element $M$ of a Noether lattice. Then the rank of $P$ is at most one.

Proof. Let $L$ denote the Noether lattice. Without any loss of generality we may suppose that $L$ is local and that $P$ is the maximal prime of $L$. For $L_{P}$ is a local Noether lattice in which the maximal prime is the congruence class of $P$. By Theorem 5.2, the rank of the congruence class of $P$ in $L_{P}$ is the same as the rank of $P$ in $L$. But if $L$ is a local Noether lattice and $P$ is the maximal prime of $L$, then $M$ is primary with $P$ as the associated prime. For let $P_{i}$ be a prime associated with a normal decomposition of $M$. Then $P \supseteqq P_{i}$ since $P$ is maximal. But since $P$ is minimal over $M$ we must have $P=P_{i}$ and hence $M$ is primary associated with $P$.

Now let $P \supset P_{1} \supseteqq P_{2}$. Then $L / P_{2}$ is a local Noether lattice with maximal prime $P$. The zero element of $L / P_{2}$ is $P_{2}$ which is prime by Lemma 4.2. Also by Lemma 4.2, the principal element $M \cup P_{2}$ of $L / P_{2}$ is primary with $P$ as associated prime. Hence by Theorem $6.3, P$ is the only nonzero prime of $L / P_{2}$. But by Lemma 4.2, $P_{1}$ is a prime of $L / P_{2}$ which is distinct from $P$. Thus $P_{1}=P_{2}$ and the rank of $P$ is at most one.

We will conclude this section by proving the theorem which extends the result of Theorem 6.4 to nonprincipal elements. A preliminary lemma is required.

Lemma 6.4. Let $L$ be a Noether lattice and let $P=P_{0} \supset P_{1} \supset \cdots$ $\supset P_{r-1} \supset P_{r}$ be a chain of prime elements of $L$. If $M$ is a principal element of $L$ such that $P \supseteqq M$, then there exists a chain of prime elements $P=P_{0}^{*} \supset P_{1}^{*} \supset \cdots P_{r-1}^{*} \supset P_{r}$ such that $P_{r-1}^{*} \supseteqq M$.

For let us set $P_{0}^{*}=P_{0}=P$. If $P_{1} \supseteqq M$ we set $P_{1}^{*}=P_{1}$, otherwise we let $P_{1}^{*}$ be a minimal prime such that $P \supseteqq P_{1}^{*} \supseteqq M \cup P_{2}$. Then $P_{1}^{*}$ is a minimal prime containing the principal element $M \cup P_{2}$ of $L / P_{2}$. Hence by Theorem 6.4 we conclude that $P \neq P_{1}^{*}$ since $P$ is of rank two in $L / P_{2}$. Also since $P_{1} \nsupseteq M$ we have $P_{2} \nsupseteq M$ and hence $P_{1}^{*} \neq P_{2}$. Thus 
$P=P_{0}^{*} \supset P_{1}^{*} \supset P_{2}$ and $P_{1}^{*} \supseteqq M$. Repeating this argument $r-1$ times gives the chain $P=P_{0} \supset P_{1}^{*} \supset \cdots \supset P_{r-1}^{*} \supset P_{r}$ with $P_{r-1}^{*} \supseteqq M$.

TheOREM 6.5. Let $L$ be a Noether lattice and let $P$ be a minimal prime containing $M_{1} \cup \cdots \cup M_{k}$ where each $M_{i}$ is principal. Then the rank of $P$ is at most $k$.

Proof. Let $P=P_{0} \supset P_{1} \supset \cdots \supset P_{r-1} \supset P_{r}$ be an arbitrary chain of primes beginning with $P$. Then $P \supseteqq M_{k}$ and hence by Lemma 6.4 there exists a chain $P=P_{0}^{*} \supset P_{1}^{*} \supset \cdots \supset P_{r-1}^{*} \supset P_{r}$ such that $P_{r-1}^{*} \supseteqq M_{k}$. Now $P$ is a minimal prime containing $\left(M_{1} \cup M_{k}\right) \cup \cdots \cup\left(M_{k-1} \cup M_{k}\right)$ where each $M_{i} \cup M_{k}$ is principal in $L / M_{k}$. Since $P=P_{0}^{*} \supset \cdots \supset P_{r-1}^{*}$ is a prime chain in $L / M_{k}$ we may suppose by induction that $r-1 \leqq k-1$ and hence conclude that $r \leqq k$. This completes the proof of the theorem.

7. Polynomial ideals. In this section it will be shown that the principal elements of the lattice of ideals of a unique factorization integral domain are precisely the principal ideals. In particular, this result holds for ideals in a polynomial ring.

THEOREM 7.1. Let $R$ be a unique factorization integral domain. Then every principal element in the lattice of ideals of $R$ is a principal ideal.

Proof. Let $M=\left(m_{1}, \cdots, m_{k}\right)$ be a principal element in the lattice of ideals of $R$. Then $M \supseteqq\left(m_{i}\right)$ and hence $\left(m_{i}\right)=\left(m_{i}\right) \cap M=\left(\left(m_{i}\right): M\right) M=$ $X_{i} M$ where $X_{i}=\left(m_{i}\right): M$. Then there exist elements $x_{i 1}, \cdots, x_{i k}$ in $X_{i}$ such that

$$
m_{i}=x_{i 1} m_{1}+\cdots+X_{i k} m_{k} \quad i=1, \cdots, k
$$

Let

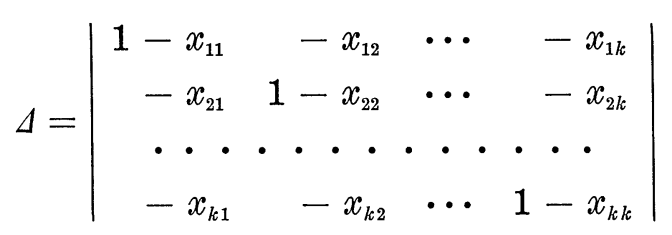

Then $\Delta m_{i}=0$ for $i=1, \cdots, k$ and hence $\Delta=0$ since $R$ is an integral domain. But $\Delta=1-x$ where $x \in X_{1} \cup \cdots \cup X_{x}$. Hence $X_{1} \cup \cdots \cup X_{k}=$ (1) and thus there exist elements $x_{i} \in X_{i}$ such that

$$
x_{1}+\cdots+x_{n}=1 \text {. }
$$

Since $\left(m_{i}\right)=X_{i} M$ there exist elements $r_{i j}$ such that

$$
x_{i} m_{j}=r_{i j} m_{i} .
$$


Let $b$ be the g. c. d. of $m_{1}, \cdots, m_{k}$. Then since $R$ is a unique factorization domain it follows that $x_{i}$ is a multiple of $m_{i} / d$. Thus $d x_{i}=y_{i} m_{i}$. Hence

$$
d=d x_{1}+\cdots+d x_{k}=y_{1} m_{1}+\cdots+y_{k} m_{k} \in M .
$$

On the other hand it is clear that $M \subseteq(d)$. Hence $M=(d)$ and it follows that $M$ is a principal ideal.

It should be noted that if $L$ is a Noether lattice which satisfies the cancellation law for nonzero elements and in which every element has a unique factorization into primes (for example, the lattice of ideals of the ring of integers in an algebraic number field), then $A: B=(A \cap B) / B$ and hence 1.1 and 1.2 hold identically in $L$. Thus every element of $L$ is principal. R. S. Pierce notes that the converse is also true. If $L$ is a Noether lattice in which 0 is prime and in which every element is principal, then the cancellation law holds in $L$ and every element of $L$ has a unique decomposition into primes. The cancellation law follows from Lemma 6.3 and the unique decomposition into primes can be deduced from Corollary 3.1.

8. Distributive lattices. Let $L$ be a distributive lattice satisfying the ascending chain condition and with a null element 0 . Then if the meet operation is taken as the multiplication it is well known (Ward [5]) that $L$ is a multiplicative lattice with residuation. Since $L$ is modular and satisfies the ascending chain condition, $L$ will be a Noether lattice if and only if the principal elements of $L$ generate $L$ under finite joins.

Lemma 8.1. $\quad M$ is a principal element of $L$ if and only if $M$ has a complement in $L$.

For let $M$ be principal. Then $0 \supseteqq(0: M) \cap M$ and hence $(0: M) \cap M=$ 0 . On the other hand, $0: M \cup M=[0 \cup(M \cap M]: M=M: M=I$ and hence $0: M$ is a complement of $M$. Conversely, suppose that $M$ is an element with a complement $M^{\prime}$. It is easily verified that $X: M=$ $X \cup M^{\prime}$. Hence

$$
\begin{gathered}
{[A \cup(B \cap M)]: M=A \cup(B \cap M) \cup M^{\prime}=A \cup M^{\prime} \cup B=A: M \cup B} \\
{[A \cap(B: M)] \cap M=\left[A \cap\left(B \cup M^{\prime}\right)\right] \cap M=(A \cap M) \cap B}
\end{gathered}
$$

and $M$ is principal. The proof of the lemma is thus complete.

Now the complemented elements of a distribution lattice are closed under meet and join and form a Boolean algebra. Thus we get

Theorem 8.1. A distributive lattice is a Noether lattice if and only if it is a finite Boolean algebra. 
It should be noted that the prime elements of a Boolean algebra are the elements covered by the unit element. Any two such elements are non comparable and hence the rank of every proper prime is zero.

\section{REFERENCES}

1. C. Chevalley, On the notion of the ring of quotients of a prime ideal, Bull. Amer. Math. Soc., 50 (1944), 93-97.

2. W. Krull, Dimensionstheorie in Stellenringe J. reine angew, Math. 179 (1938), 204-226.

3. D. Rees, Two classical theorems of ideal theory, Proc. Camb. Phil. Soc., 52 (1956), 155-157.

4. M. Ward and R. P. Dilworth, Residuated lattices, Trans. Amer. Math. Soc., 45 (1939), 335-354.

5. M. Ward, Residuated distributive lattices, Duke Math. Journal 6 (1940) $641-651$.

CALIFornia Institute of Technology 


\title{
PACIFIC JOURNAL OF MATHEMATICS
}

\author{
EDITORS
}

Ralph S. Phillips

Stanford University

Stanford, California

M. G. Arsove

University of Washington

Seattle 5, Washington
A. L. Whiteman

University of Southern California Los Angeles 7, California

Lowell J. Paige

University of California

Los Angeles 24, California

\section{ASSOCIATE EDITORS}
E. F. BECKENBACH
D. DERRY
M. OHTSUKA
H. L. ROYDEN
E. SPANIER
E. G. STRAUS
T. M. CHERRY
F. WOLF

\section{SUPPORTING INSTITUTIONS}

\author{
UNIVERSITY OF BRITISH COLUMBIA \\ CALIFORNIA INSTITUTE OF TECHNOLOGY \\ UNIVERSITY OF CALIFORNIA \\ MONTANA STATE UNIVERSITY \\ UNIVERSITY OF NEVADA \\ NEW MEXICO STATE UNIVERSITY \\ OREGON STATE UNIVERSITY \\ UNIVERSITY OF OREGON \\ OSAKA UNIVERSITY \\ UNIVERSITY OF SOUTHERN CALIFORNIA
}

\author{
STANFORD UNIVERSITY \\ UNIVERSITY OF TOKYO \\ UNIVERSITY OF UTAH \\ WASHINGTON STATE UNIVERSITY \\ UNIVERSITY OF WASHINGTON \\ $*$
AMERICAN MATHEMATICAL SOCIETY \\ CALIFORNIA RESEARCH CORPORATION \\ SPACE TECHNOLOGY LABORATORIES \\ NAVAL ORDNANCE TEST STATION
}

Mathematical papers intended for publication in the Pacific Journal of Mathematics should be typewritten (double spaced), and the author should keep a complete copy. Manuscripts may be sent to any one of the four editors. All other communications to the editors should be addressed to the managing editor, L. J. Paige at the University of California, Los Angeles 24, California.

50 reprints per author of each article are furnished free of charge; additional copies may be obtained at cost in multiples of 50 .

The Pacific Journal of Mathematics is published quarterly, in March, June, September, and December. Effective with Volume 13 the price per volume (4 numbers) is $\$ 18.00$; single issues, $\$ 5.00$. Special price for current issues to individual faculty members of supporting institutions and to individual members of the American Mathematical Society: $\$ 8.00$ per volume; single issues $\$ 2.50$. Back numbers are available.

Subscriptions, orders for back numbers, and changes of address should be sent to Pacific Journal of Mathematics, 103 Highland Boulevard, Berkeley 8, California.

Printed at Kokusai Bunken Insatsusha (International Academic Printing Co., Ltd.), No. 6, 2-chome, Fujimi-cho, Chiyoda-ku, Tokyo, Japan.

PUBLISHED BY PACIFIC JOURNAL OF MATHEMATICS, A NON-PROFIT CORPORATION

The Supporting Institutions listed above contribute to the cost of publication of this Journal, but they are not owners or publishers and have no responsibility for its content or policies. 


\section{Pacific Journal of Mathematics}

\section{Vol. 12, No. $2 \quad$ February, 1962}

William George Bade and Robert S. Freeman, Closed extensions of the Laplace operator determined by a general class of boundary conditions . . . . . . . 395

William Browder and Edwin Spanier, H-spaces and duality ............. 411

Stewart S. Cairns, On permutations induced by linear value functions . . . . . . . 415

Frank Sydney Cater, On Hilbert space operators and operator roots of

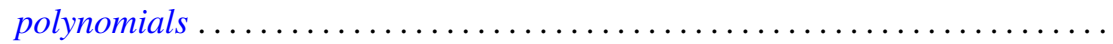

Stephen Urban Chase, Torsion-free modules over $K[x, y] \ldots \ldots \ldots \ldots \ldots \ldots \ldots 437$

Heron S. Collins, Remarks on affine semigroups . . . . . . . . . . . . . . . . 449

Peter Crawley, Direct decompositions with finite dimensional factors . . . . . . . 457

Richard Brian Darst, A continuity property for vector valued measurable

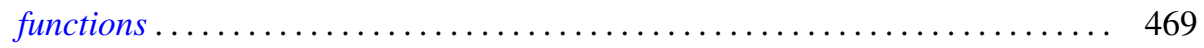

R. P. Dilworth, Abstract commutative ideal theory ................. 481

P. H. Doyle, III and John Gilbert Hocking, Continuously invertible spaces . . . . . . 499

Shaul Foguel, Markov processes with stationary measure . . . . . . . . . . . 505

Andrew Mattei Gleason, The abstract theorem of Cauchy-Weil ............ 511

Allan Brasted Gray, Jr., Normal subgroups of monomial groups . . . . . . . . . . 527

Melvin Henriksen and John Rolfe Isbell, Lattice-ordered rings and function

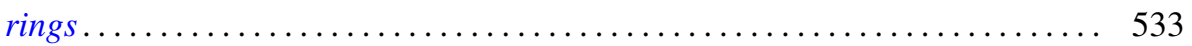

Amnon Jakimovski, Tauberian constants for the $[J, f(x)]$ transformations . ..... 567

Hubert Collings Kennedy, Group membership in semigroups . . . . . . . . . . . 577

Eleanor Killam, The spectrum and the radical in locally $m$-convex algebras ..... 581

Arthur H. Kruse, Completion of mathematical systems . . . . . . . . . . . . . 589

Magnus Lindberg, On two Tauberian remainder theorems ................ 607

Lionello A. Lombardi, A general solution of Tonelli's problem of the calculus of

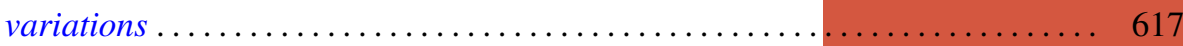

Marvin David Marcus and Morris Newman, The sum of the elements of the powers

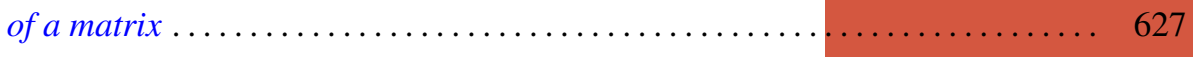

Michael Bahir Maschler, Derivatives of the harmonic measures in

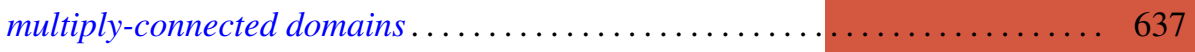

Deane Montgomery and Hans Samelson, On the action of $\mathrm{SO}(3)$ on $S^{n} \ldots \ldots \ldots 649$

J. Barros-Neto, Analytic composition kernels on Lie groups . . . . . . . . . . . . 661

Mario Petrich, Semicharacters of the Cartesian product of two semigroups ...... 679

John Sydney Pym, Idempotent measures on semigroups . . . . . . . . . . . . 685

K. Rogers and Ernst Gabor Straus, A special class of matrices . . . . . . . . . . . . 699

U. Shukla, On the projective cover of a module and related results . . . . . . . . . 709

Don Harrell Tucker, An existence theorem for a Goursat problem . . . . . . . . . . . 719

George Gustave Weill, Reproducing kernels and orthogonal kernels for analytic

differentials on Riemann surfaces ......................... 729

George Gustave Weill, Capacity differentials on open Riemann surfaces ........ 769

G. K. White, Iterations of generalized Euler functions . . . . . . . . . . . . . 777

Adil Mohamed Yaqub, On certain finite rings and ring-logics . . . . . . . . . 785 